\title{
Identidades y preferencias lingüísticas en comunidades de la Selva Central del Perú
}

\author{
Identities and Linguistic Preferences in Selva Central Communities
}

\author{
Pedro Manuel Falcón Ccenta \\ Universidad Nacional Mayor de San Marcos, Lima, Perú \\ Contacto: pfalconc@unmsm.edu.pe \\ https://orcid.org/0000-0002-0863-5735
}

\section{Resumen}

El propósito del estudio es analizar, desde la perspectiva teórico-mentalista, las preferencias lingüísticas de los pobladores indígenas bilingüesamazónicos en términos afectivos, cognoscitivos y socioculturales. Para ello, se evalúan los factores como edad, sexo, escolaridad y procedencia que condicionan las actitudes positivas o negativas hacia las lenguas originarias y hacia el castellano. Estos factores, además, comprometen las identidades, los niveles de autoestima y los soportes socioculturales. Asimismo, en este contexto permiten comprender con mayor objetividad la predisposición valorativa de los usuarios indígenas respecto a su propia lengua y hacia el castellano en conexión con los elementos de identificación sociocultural o el distanciamiento con su grupo que, sin duda, comprometen seriamente la vulnerabilidad de las lenguas.

Palabras clave: Identidad; Actitudes lingüísticas; Amazonía; Sociolingüística

\begin{abstract}
The purpose of the study is to analyze from the mentalist theoretical perspective, the languages preferences of the indigenous bilingual Amazonian settlers in affective, cognitive and sociocultural terms. For this, the factors age, sex, schooling and origin are evaluated that condition the positive or negative attitudes towards the native languages and towards Spanish. In addition, these factors compromise the identities, levels of self-esteem, socio-cultural supports. It also allows, through the evaluation of the variables of influence in the languages preferences of the Amazonian bilingual populations, to understand more objectively the value of indigenous users in relation to their own language and to Spanish in connection with elements of socio-cultural identification or distancing themselves from their group that with no doupt, compromises the vulnerability of the involved languages.
\end{abstract}

Keywords: Identity; Linguistic attitudes; Bilingual Amazonian: Sociolinguistic 


\section{Introducción}

La evaluación de las preferencias lingüísticas en indígenas bilingües (nomatsigenga, yanesha y ashaninka) se sustenta en las dimensiones afectivas, cognoscitivas y socioculturales. Para ello, se echa mano de técnicas de encuesta y observación que se viabilizan con el auxilio de los instrumentos de recolección de datos (IRD) adecuados a las características socioculturales de la población de estudio (Vargas, 2017). De esta manera, se describen los diferentes contextos comunicativos y las preferencias de lenguas en correlación con las variables de edad, sexo, escolaridad y procedencia. Es pertinente señalar que las preferencias lingüísticas guardan estrecha relación con la construcción de la identidad. Al respecto, "se puede distinguir conceptualmente identidad a nivel individual y a nivel colectivo que necesariamente están implicados, pues los conocimientos y experiencias del individuo cobran sustento en tanto sujeto involucrado en la red sociocultural" (Howard, 2007, p. 43); así, el comportamiento actitudinal de los individuos y las funciones que tienen en la sociedad comprometen rasgos identitarios.

El estudio tomó como base metodológica la evaluación de los "pares ocultos o pares falsos" y la observación participativa. En relación con la primera técnica, se elaboró el instrumento de "los pares falsos", el mismo que se complementó con la técnica del diferencial semántico. Al respecto, se pidió a los colaboradores seleccionados que escuchen emisiones grabadas de bilingües coordinados en lengua originaria y castellana, luego puntúen características como inteligencia, simpatía, etc., que se suponen en el emisor. Se trata de una técnica de medición que permite exponer los sentimientos más íntimos del que escucha y de las actitudes estereotipadas hacia los grupos cuya lengua es distintiva (Gómez, 1998, p. 54). Finalmente, se diseñaron las estrategias para la implementación de la técnica participativa durante el proceso de trabajo de campo.

Según los objetivos y las variables edad, sexo, escolaridad y procedencia, se aplicó la técnica de "los pares falsos" a 82 colaboradores ashaninka; a 88 colaboradores yanesha y a 87 colaboradores nomatsigenga. Los resultados cuantitativos, en términos generales, señalan una relación afectiva con la lengua originaria, especialmente por 
parte de los adultos y adultos mayores; cualitativamente se advierte en los jóvenes ashaninka y yanesha, en el orden señalado, una orientación más intensa hacia el uso del castellano, lo que evidencia rasgos de un proceso acelerado de desplazamiento de lengua originaria y pérdida sistemática de la identidad lingüístico-cultural. En el caso de los nomatsigenga, existe una mayor identificación con la lengua y cultura por parte de la población, incluidos los jóvenes, aunque con mayor exposición al castellano respecto de los mayores.

En el apartado 1 se discutirán los enfoques teóricos a fin de contextualizar la perspectiva que respalda el trabajo. En el apartado 2 se describe la metodología para enmarcar el tipo de investigación y las características detalladas de la organización del trabajo empírico, el uso de las técnicas y los protocolos correspondientes. En el apartado 3 se presentan los resultados y la evaluación cualitativa y cuantitativa. Finalmente, en el apartado 4, se desarrolla la interpretación y discusión de los resultados.

\section{Marco teórico referencial}

"La identidad supone una alteridad y bien podrían considerarse a ambas como caras de una misma moneda" (Álvarez, Martínez \& Urdaneta, 2001, p. 146). Por ello, se asume que la identidad se refleja en las actitudes de los individuos, en la interiorización de las reacciones de los demás, muchas veces negativas y desfavorables para los usuarios de las lenguas originarias. Así, los problemas rebasan la propia condición lingüística y se relacionan más bien con una situación de lenguas en contacto y problemas de bilingüismo que inevitablemente comprometen caracteres sociales de alienación y transculturación (Alvar López, 1986, p. 12).

Sarnoff afirmó que un comportamiento actitudinal es una "disposición a reaccionar favorable o desfavorablemente a una serie de objetos" (1996, p. 279). Después de esta primera aproximación teórica, se trabajaron conceptos en busca de una descripción integral de la naturaleza de las actitudes lingüísticas.

El estudio sobre actitudes lingüísticas ha cobrado mucho interés, pues no solo compromete la identidad lingüístico-cultural, sino puede contribuir 
poderosamente a la difusión de cambios lingüísticos. La producción de usuarios de una lengua desfavorecida en una comunidad de habla con una actitud negativa, puede proyectarse en la siguiente generación (Carranza Romero, 1982, p. 63).

Las actitudes son manifestaciones valorativas que proyectan la percepción que se tiene no solo hacia las variedades de una lengua o hacia lenguas distintas, sino, de manera sustantiva, también hacia las personas que las hablan, por lo que su estudio proporciona información para entender las relaciones interculturales (Castillo Hernández, 2007, p. 26). Por ello, compromete no solamente actitudes vinculadas a las lenguas, sean L1 y L2, a los usuarios o a la identificación con sus grupos, sino además al proceso mismo de la formación escolar y su integración con la sociedad hegemónica. Fasold afirmó: “A menudo las actitudes lingüísticas son el reflejo de actitudes hacia miembros de grupos étnicos diferentes" (1996, p. 231). En tal sentido, involucra extensionalmente aspectos relativos al proceso de escolaridad; evidentemente compromete niveles de autoestima, soportes socioculturales y lingüísticos, al igual que redes de interacción social.

En la medida en que las actitudes se adquieren en el marco de un contexto sociocultural, no necesariamente son duraderas y estables; pueden variar dependiendo de las motivaciones psicosociolingüísticas, pues involucran un juicio frente a la forma de habla usada, frente a los hablantes, frente a sus comportamientos lingüísticos y a los símbolos o referentes que esas formas de habla o comportamientos crean.

La conciencia sociolingüística presupone una estrecha relación con las actitudes lingüísticas que facultan a los usuarios de una comunidad estructurar un continuum de valoraciones positivas o negativas en torno a los hechos lingüísticos o hacia las lenguas de convivencia. En esta orientación "parece existir una estrecha correlación entre conciencia y factor sociocultural, frecuentemente cruzado con el sexo de los hablantes" (García Marcos, 2015, p. 89).

\subsection{El enfoque mentalista}

Los defensores del enfoque conductista ven un elemento único, el cual generalmente está relacionado a menudo con lo afectivo o lo valorativo. 
Entonces, en la medida en que una de las líneas de evaluación de actitudes está relacionada con el contacto lingüístico, la selección e influencia de lenguas que pueden trastocar no solo la identidad sino fomentar la discriminación lingüística, se asume que el enfoque mentalista conducirá al análisis de componentes diversos de manera más profunda y detallada.

Con base en las referencias de Rebecca Agheyisi y Joshua Fishman (1970, p. 138), Blas sostuvo que "la actitud es entendida como una variable que interviene entre un estímulo que afecta a la persona y la respuesta que se desprende de este" (2012, p. 322); asimismo, desde la óptica psicosociolingüística se asume como un estado interno del individuo, una disposición mental hacia unas condiciones o unos hechos sociolingüísticos concretos. En esos términos, la actitud es considerada como "la relación entre un estímulo que afecta a un sujeto y la respuesta de este sujeto, como una categoría intermedia entre el estímulo y el comportamiento individual" (Gómez Molina, 1998, p. 27). Por ello, debe entenderse meridianamente la importancia del empleo de las estrategias metodológicas que posibiliten acceder con mayor precisión a la estructura componencial de las actitudes del individuo.

Las actitudes lingüísticas no responden a consideraciones biológicas, pues no son transferidas genéticamente; son predisposiciones que se construyen en el marco del desarrollo sociocultural y tienden a ser relativamente estables en el tiempo, dependiendo de factores de influencia. La actitud compromete la presencia de elementos o subcomponentes: una valoración (componente afectivo), un saber o creencia (componente cognoscitivo) y una conducta (componente conativo). Entre los estudiosos mentalistas existen oposiciones respecto de cómo se relacionan los elementos mencionados. Por esto, es necesario plantear el problema relativo a describir la estructura componencial de las actitudes lingüísticas.

Las propuestas psicosociológicas más trabajadas y conocidas pertenecen, entre otros, a Fishbein (1965) para quien las lenguas, las situaciones o los hechos lingüísticos dan lugar a las actitudes y a las creencias. La naturaleza afectiva es el único componente que forma las actitudes, porque se fundamentan en la valoración subjetiva y sentimental respecto de un objeto. En un plano diferente, 
las creencias se forman por un componente cognoscitivo y un componente de acción o de conducta. En esta perspectiva considera que las actitudes y creencias tienen lugar en dos dimensiones diferentes (Fishbein, 1965, p. 119).

Otros investigadores dan más relevancia al dinamismo del modelo que a la relación estructural de sus componentes. Por ejemplo, Moreno Fernández (2009), apoyado en Street y Hooper (1982), planteó un modelo de valoración del habla. Este se basa en los juicios de valor y en los usos lingüísticos de los interlocutores. En este caso, los procesos cognoscitivos y de conducta están determinados por tres valores: a) los conocimientos recibidos y los prejuicios de los hablantes (estereotipos, procesamiento de la información, características de la personalidad, expectativa sociológica); b) las características del habla, del mensaje (acento, dialecto, elementos paralingüísticos); c) las intenciones de los interlocutores (Moreno Fernández, 2009, pp. 181-182).

En el marco de esta perspectiva, el comportamiento actitudinal de los bilingües de la Selva Central hacia la lengua originaria y hacia el castellano compromete rasgos identitarios, esto es, la disposición mental reflejada en dimensiones cognitivas, afectivas y conductuales hacia las lenguas involucradas, las mismas que estarán acompañadas de contextos socioculturales que se expresarán a través de relaciones interculturales: intracomunal, intercomunal y extracomunal. En dicho entorno se producirán los usos y funciones de las lenguas en contacto, en estrecha relación con las manifestaciones de identidad lingüística y cultural.

\subsection{Identidad lingüística}

La evaluación de las actitudes lingüísticas compromete rasgos de identidad lingüística y cultural. Los grupos sociales adoptan determinadas actitudes hacia otros grupos o comunidades de habla, según las diferencias que se establezcan dentro de la sociedad; además, estas actitudes influyen en los modelos culturales que caracterizan a estos grupos, incluyendo la lengua. "Si hay una relación intensa entre lengua e identidad, esta relación debería manifestarse en las actitudes de los individuos" (Appel \& Muysken, 1996, p. 30). De esta manera, esta relación involucra valoraciones positivas o negativas hacia los grupos con una identidad determinada. 
Los objetivos principales del estudio sobre actitudes lingüísticas son lo que piensan los hablantes sobre las lenguas, es decir, lo que piensan dichos individuos sobre los hablantes de esas lenguas (Blas Arroyo, 2012, p. 322). Esgrimiendo estos considerandos, se puede explicitar que la valoración hacia la lengua originaria es negativa, especialmente por parte de los usuarios jóvenes de la lengua ashaninka; por ello, las actitudes desfavorables en acciones de cotidianidad comprometen no solo la valoración y la identidad con la lengua y la cultura, sino también con el futuro de estas.

La lealtad lingüística tiene mucho que ver con la identidad de ciertas comunidades de habla, las cuales no solo prefieren utilizar la variante propia, sino que juzgan de manera menos positiva y hasta rechazan las variantes o lenguas foráneas. Sin embargo, suele ocurrir un fenómeno contrario que se evidencia en conductas y usos de la lengua originaria y castellano en desigualdad funcional y afectiva, especialmente en jóvenes ashaninka que ponderan el castellano con matices solapadamente discriminatorios respecto de la población que se identifica mucho más con la lengua originaria (adultos mayores).

Según Appel y Muysken, "la identidad de un grupo se suele calificar como identidad cultural o étnica, o bien, como su etnicidad" (1996, p. 24). Esto comprende que hay una relación muy estrecha entre la identidad y el etnocentrismo de un grupo. Así, la identidad constituye un conjunto de rasgos que particularizan a cada comunidad de habla y la diferencia de otra.

En el estudio, se asume en primer lugar que la lengua se solapa con la cultura como rasgos particulares de una comunidad de habla o grupo étnico; en segundo lugar, los escenarios interculturales y la relación que se establece entre una lengua sociopolíticamente dominante y una originaria, en situación de diglosia, afecta lingüística, sociocultural y emocionalmente a los hablantes indígenas; por ello, se vincula con las actitudes lingüísticas que pueden manifestarse como negativas o positivas, sea desde dimensiones afectivas, cognoscitivas o conductuales. 


\subsection{Factores sociolingüísticos}

\subsubsection{Sexo}

Si bien en décadas pasadas se le ha atribuido un peso sustantivo en la variación lingüística estableciendo un mayor conservadurismo en las mujeres respecto de los hombres, actualmente no puede establecerse esa distancia; los hombres son también sensibles a cierto estatuto de prestigio, sobre todo cuando intersectamos con aspectos como las actitudes y la conciencia sociolingüística; por ello, el sexo es un factor de segundo orden, como algo que puede subordinarse a otras dimensiones sociales con mayor poder de determinación (López Morales, 2015, p. 220). En el marco de la investigación en las comunidades yanesha, nomatsigenga y ashaninka, si bien esta variable no tiene mayor repercusión en la evaluación cuantitativa, adquiere importancia en la evaluación cualitativa, pues en correlación con la variable edad evidencian en la mujer rasgos relativamente de mayor conservadurismo en comparación con los varones.

\subsubsection{Escolaridad}

"La sociolingüística [...] ha comprobado que el nivel educativo de los hablantes determina de forma directa y clara la variación lingüística: es normal que las personas más instruidas hagan mayor uso de las variantes que son consideradas como más prestigiosas o que más se ajustan a la norma" (Moreno Fernández, 2009, p. 61). La variable grado de instrucción implica el nivel de escolaridad que ha sido alcanzado por las personas que conforman la muestra y en qué medida influye en las actitudes lingüísticas hacia los idiomas, la lengua originaria o castellano. En tal sentido, los pobladores ashaninka de mayor instrucción (jóvenes) no solo generan variaciones y eventualmente cambios en el sistema de la lengua, sino que muchos de ellos contribuyen en el sistemático proceso de precarización de la originaria y una notoria alta valoración (especialmente cognoscitiva) del castellano; mientras que los pobladores nomatsigenga y yanesha evidencian afectivamente mayor valoración hacia la lengua nativa. 


\title{
1.3.3. Edad
}

Esta variable adquiere significativa importancia en los procesos de interacción comunicativa, pues socioculturalmente cumple un rol fundamental en diversas sociedades a tal punto que influye no solo en el habla, en la valoración de las lenguas, sino también en la organización social de una comunidad.

\begin{abstract}
Para la sociolingüística, la edad no es simplemente un factor cronológico sino que lleva consigo toda una serie de implicaciones sociales, sicológicas y económicas: además de ser un factor que determina cambios de conducta social y lingüística, hasta el punto de que se le da gran importancia a las variables que adopta la lengua dentro de los distintos grupos de edad. (Areiza, Cisneros \& Tabares, 2012, p. 50)
\end{abstract}

En esta óptica, los indígenas yanesha, nomatsigenga y ashaninka que reflejan rasgos de valoración afectiva más intensa o menos intensa, dependiendo de la lengua originaria son los adultos (de 31 a 45 años), en menor proporción, y el grupo etario de 46 a más años, en mayor proporción.

\subsubsection{Procedencia}

En concordancia con los estudios, entre otros, de López (2015), García (2015), Moreno (2009), la procedencia geográfica responde a una variable pertinente para la evaluación sociolingüística. Específicamente se pueden vincular las actitudes indígenas bilingües amazónicas con la variable procedencia geográfica y sociocultural, pues involucra comunidades nativas con mayor o menor interacción con agentes foráneos de diferente orden cuya ascendencia compromete rasgos actitudinales hacia las lenguas de convivencia.

\section{Metodología y técnicas de investigación}

En el marco de la metodología propuesta, de manera complementaria, se utilizaron los métodos directo e indirecto, cuyas técnicas fueron elaboradas en función a la realidad sociocultural de la zona a fin de desarrollar con la mayor claridad y objetividad un análisis cualitativo y cuantitativo de los datos. 
La elaboración del cuestionario posibilitó la medición de las variables inherentes: sexo, edad, y la variable adscrita: escolaridad. La técnica de "apareamiento disfrazado" o "pares falsos" utilizada en varios estudios como el realizado por Silva-Corvalán (2001) en donde se recurre a un mismo hablante para realizar dos grabaciones, cada una de ellas en una lengua diferente con el fin de que el oyente tenga la impresión de que escucha a dos personas diferentes y someta sus evaluaciones en esas condiciones (Silva-Corvalán, 2001, p. 69). Esta técnica, consecuentemente, permite que se obtengan datos de valoración hacia la lengua y no hacia la persona que la habla; por ello, para la grabación de dos textos, se eligió a dos bilingües coordinados. Cada uno de los bilingües en lengua originaria-castellano seleccionados generó dos audios; uno en lengua originaria (yanesha, ashaninka, nomatsigenga) y otro en castellano haciendo un total de cuatro audios para cada caso, los mismos que se intercalaron a fin de generar en el oyente la impresión de estar oyendo a cuatro personas, dos hablantes del castellano y dos hablantes de la lengua originaria correspondiente y propiciar la evaluación de las actitudes lingüísticas a través de la evaluación de los hablantes.

La técnica del "diferencial semántico" consiste en presentar términos dicotómicos opuestos con varias posibilidades intermedias (generalmente entre cinco a siete). Así, se le pide al colaborador que marque una puntuación en el espacio que mejor considere para determinar a cuál se acerca más el hablante, según la opinión del entrevistado. Para ello, se propuso doce pares de adjetivos que se miden de acuerdo con una escala ordinal y están referidos a características que posibilitan la evaluación sociocultural y laboral de los productores de los audios. Estos pares se miden en una escala del uno al cinco, siendo uno la más negativa y cinco la más positiva.

Asimismo, los pares de adjetivos se distribuyeron en dos grupos:

i) Características personales y juicios sobre la percepción de la persona: malobueno, flojo-trabajador, orgulloso-humilde, débil-fuerte, triste-alegre, pobre-rico, aburrido-divertido, chusco-fino. 
ii) Características relacionadas al ámbito laboral: inseguro-segura de sí, tontointeligente, mentiroso-sincero, maleducado-educado.

Complementariamente se elaboraron tres preguntas semiabiertas para demostrar la aceptación de servicios laborales y/o profesionales; es decir, se preguntó directamente a los encuestados si ellos contratarían los servicios de los sujetos que realizaron las grabaciones para determinadas labores. Las preguntas fueron las siguientes:

i) ¿Solicitarías apoyo a esta persona (de habla castellana/lengua originaria) para planificar la fiesta de tu comunidad?

ii) ¿Te gustaría que esta persona te diera clases de castellano/lengua originaria?

iii) ¿Dejarías que esta persona organice las actividades de la comunidad?

Finalmente, se diseñaron las estrategias para la implementación de la técnica participativa durante el proceso de trabajo de campo; se estructuró una guía de observación y un cuadro codificado por familias a fin de acceder sistemáticamente al levantamiento de datos e información (situaciones comunicativas, como asambleas comunales, actividades, etc., en las que se emplean el castellano y la lengua originaria) sin interferir con las actividades cotidianas de los indígenas de las comunidades seleccionadas.

La metodología tuvo como soporte el diseño mixto, ya que se combinó técnicas cuantitativas y cualitativas, siendo este tipo de métodos enmarcados en la tipología metodológica mixta. Es decir, "las ideas constituyen el primer acercamiento a la realidad objetiva (desde la perspectiva cuantitativa), a la realidad subjetiva (desde la aproximación cualitativa) o a la realidad intersubjetiva (desde la aproximación mixta)" (Hernández, Fernández \& Baptista, 2014, p. 24).

\subsection{Tipo de investigación}

El diseño de esta investigación es correlacional. "[E]stos diseños describen relaciones entre dos o más categorías, conceptos o variables en un momento 
determinado. A veces, únicamente en términos correlacionales, otras en función de la relación causa-efecto (causales)" (Hernández, Fernández \& Baptista, 2014, p. 157). Especificando el diseño de la investigación, el diseño fue transeccional porque se trabajó en un tiempo único y es correlacional porque solo se ciñe a ordenar las variables de investigación, sin pretender establecer relaciones de causa-efecto.

Con respecto a los instrumentos y su aplicación, se procesaron los datos cuantitativos en hojas de cálculo Excel para que se pueda exportar al programa SPSS, y consecuentemente determinar las correlaciones correspondientes acorde con los objetivos del estudio.

\subsection{Población y muestra}

En el estudio, la muestra está constituida por 82 personas para el caso ashaninka, 88 para el caso yanesha y 87 para el caso nomatsigenga. Todas ellas mayores de 16 años, con competencia en la lengua originaria y el castellano, además haber nacido o vivir más de 5 años en la comunidad y que estén dentro de ella más del $60 \%$ de tiempo al año. Para la muestra se tuvo en cuenta los criterios del muestreo no probabilístico. En esta óptica, "[1]as muestras no probabilísticas o dirigidas son de gran valor, pues logran obtener los casos (personas, objetos, contextos, situaciones) que interesan al investigador y que llegan a ofrecer a una gran riqueza para la recolección y el análisis de los datos" (Hernández, Fernández \& Baptista, 2014, p. 190).

Se utilizaron técnicas de la encuesta y observación participativa, las mismas que se instrumentalizaron a través de la bitácora y guía codificada para el primer caso, y de entrevistas y cuestionarios, para el segundo caso; asimismo, se apeló a los "pares falsos" en complemento con la técnica del diferencial semántico. La técnica de la observación empleada fue inicialmente no estructurada, común en estudios de sociolingüística, pero organizada por medio de una guía y un cuadro codificado a fin de obtener información mucho más organizada; se elaboró un cuadro codificado con cinco ejes, descrito líneas arriba, que posibilitó una mayor riqueza en el registro de información cualitativa. 


\subsection{Recolección de la información}

La recolección de la información se realizó a través de la técnica matched guise; para ello, los colaboradores seleccionados escucharon cuatro audios producidos por dos bilingües coordinados en lengua originaria (yanesha, ashaninka o nomatsigenga, según corresponda) y castellano para que apliquen la calificación correspondiente del 1 al 5 a cada uno de los emisores de pares de adjetivos propuestos (ejemplo: inseguro/seguro); asimismo, se aplicó el cuestionario con preguntas cerradas y abiertas, complementadas con datos recogidos por medio de la observación participativa. Los colaboradores que conformarían la muestra tenían mínimamente que comprender la lengua originaria y la lengua castellana como principal requisito.

\section{Análisis de datos}

En el contexto inicial ya referido, los objetivos principales del estudio son: (i) plantear, por medio del cuestionario y la técnica conocida como matched guise, un análisis de las actitudes lingüísticas de los hablantes ashaninka, yanesha, nomatsigenga hacia las dos lenguas de uso habitual en la comunidad de habla, lengua originaria y castellano, en el contexto de su histórica situación de diglosia; y (ii) situar la posible incidencia de algunos factores sociales en la configuración de estas actitudes, especialmente por lo que respecta a la edad, sexo, escolaridad y procedencia con el objeto de confirmar el desplazamiento de la lengua y el debilitamiento de la identidad cultural en una perspectiva de sustitución lingüística.

\subsection{Resultados}

En términos generales, para la población ashaninka el vínculo sentimental y de identificación para con la lengua originaria parece no ser homogéneo dentro del grupo (Falcón \& Mamani, 2017). En las visitas realizadas a la comunidad de Bajo Chirani se pudo notar que hay una actitud positiva hacia el castellano, muchas veces en desmedro de la propia lengua nativa. En varias comunidades del valle del Perené, en concordancia con los estudios de Trudell (2008, p. 135), se advierte que los niños tienen como L1 el castellano y desplazan sistemáticamente la lengua originaria. 
La población yanesha, sin embargo, expresa actitud positiva hacia la lengua originaria, aunque en la cotidianidad no se evidencia la práctica de la lengua indígena, especialmente por parte de los jóvenes; finalmente, la población nomatsigenga es la más consistente no solo por el lazo sentimental hacia la lengua originaria, sino por el uso recurrente de la misma, especialmente, en ámbitos locales.

De los 82 pobladores hablantes de ashaninka, se observa que 1 persona tiene una actitud lingüística muy negativa $(1,2 \%) ; 7$ personas, negativa $(8,5 \%) ; 30$ personas, positiva $(36,6 \%)$ y 44 personas, muy positiva $(53,7 \%)$. Se deduce que los hablantes de ashaninka tienen un porcentaje alto de actitud lingüística muy positiva hacia el castellano. Respecto a la lengua originaria, se observa que 1 persona tiene una actitud lingüística muy negativa $(1,2 \%) ; 6$ personas, negativa (7,3 \%); 31 personas, positiva (37,8\%) y 44 personas, muy positiva (53,7\%). Así, se deduce que los hablantes de ashaninka en alto porcentaje tienen una actitud lingüística positiva y muy positiva hacia la lengua originaria.

Para el caso yanesha, de los 88 hablantes se observa que 8 personas tienen una actitud lingüística negativa $(9,1 \%) ; 62$ personas, positiva $(70,5 \%)$ y 18 personas, muy positiva (20,5\%). Así, según las entrevistas realizadas, la mayoría de hablantes manifiesta una actitud positiva hacia el castellano. Respecto a la lengua originaria, se observa que 1 persona tiene una actitud lingüística negativa $(1,1 \%) ; 50$ personas, positiva $(56,8 \%)$ y 37 personas muy positiva $(42 \%)$. De esta manera, se supondría que la mayoría de los hablantes tiene una actitud lingüística positiva y muy positiva hacia la lengua originaria. Según lo recabado en las entrevistas, la mayoría de hablantes afirma que la lengua yanesha es muy bonita y agradable, además de que es prestigioso hablar yanesha dentro y fuera de la comunidad, ya que esto les permite acceder a beneficios educativos.

De los 87 pobladores hablantes nomatsigenga, se observa que 1 persona tiene una actitud lingüística muy negativa $(1,1 \%) ; 5$ personas, negativa $(8,75$ $\%) ; 38$ personas, positiva (43,68 \%) y 43 personas tienen una actitud lingüística muy positiva $(49,43 \%)$. Así se deduce que los hablantes nomatsigenga cuantitativamente tienen un porcentaje alto de actitud lingüística muy positiva hacia la lengua castellana. Asimismo, se observa, respecto a la lengua originaria 
que 4 personas tienen una actitud lingüística negativa (4,6 \%); 40 personas, positiva (45,98\%), y 43 personas, muy positiva (49,43\%). Así se deduce que los hablantes nomatsigenga tienen un porcentaje alto de actitud lingüística positiva y muy positiva hacia la lengua originaria.

La información cuantitativa pondera ampliamente la lengua castellana como lengua de prestigio y proyección cognoscitiva en el desarrollo académico y laboral de las poblaciones mencionadas. Desde un enfoque cualitativo, las actitudes que expresan la población ashaninka respecto de cada una de las lenguas y los contextos de uso nos remiten a la conclusión de una mayor identificación con la lengua castellana en términos cognoscitivos y socioculturales; asimismo, el grado de vínculo afectivo con la lengua originaria es relativo, pues muchos de los jóvenes ashaninka asumen que es una lengua de los antepasados y que ahora solo la usan los viejos, aun cuando en las respuestas a los cuestionarios aplicados, caso "pares ocultos", expresan una relativa consistencia en la identificación con la lengua nativa. Con características similares aunque con mayor identificación afectiva hacia la lengua originaria, la población yanesha muestra una actitud positiva o muy positiva hacia la lengua castellana, la misma que se consolida debido a que la mayoría de las personas hablan frecuentemente castellano; además, aun cuando no la usen frecuentemente, los jóvenes afirman que la lengua yanesha es muy bonita y agradable, es prestigiosa dentro y fuera de la comunidad, ya que esta proyección les permite acceder a oportunidades y beneficios educativos que gradualmente se van concretando.

En el caso de la población nomatsigenga, si bien se pondera la lengua castellana, no se advierte rasgos que signifiquen deslealtad lingüística a la lengua originaria. Con mayor intensidad respecto de los grupos ashaninka y yanesha evidencian marcada identificación lingüística y cultural, especialmente en la comunidad de Alto Anapati, pues en el marco de la cotidianidad se hace uso de la lengua a escala intracomunal, en diferentes contextos, incluido, el hogar.

En suma, en situaciones pragmáticas y de interacción natural entre pares y con foráneos, la actitud lingüística hacia la lengua originaria de los adultos y mayores adultos es medianamente positiva desde la dimensión afectiva, mientras que 
desde la dimensión cognoscitiva y sociocultural es negativa, especialmente entre los jóvenes. De esta manera, de acuerdo con los datos recogidos, se aprecia una actitud positiva hacia el castellano con matices poco consistentes en el ámbito intracomunal de desplazamiento sistemático de su lengua en favor del castellano para los casos ashaninka y yanesha.

En el análisis cualitativo, respecto de la actitud lingüística hacia la lengua originaria de acuerdo con las variables del estudio, podemos caracterizar los factores que condicionan la predisposición valorativa de los usuarios indígenas nomatsigenga respecto a su lengua, al castellano y a su identificación con el grupo y la sociedad en su conjunto. La mayoría de los grupos etarios (16-30 y 31-45), y en mayor proporción de 46 años a más en el desarrollo de actividades cotidianas ponderan el uso de la lengua nomatsigenga; en situaciones pragmáticas y de interacción natural entre pares, la actitud lingüística hacia la lengua originaria de los adultos y adultos mayores es preponderantemente positiva desde la dimensión afectiva, mientras que desde la dimensión cognoscitiva y sociocultural se pondera la lengua castellana.

\subsubsection{Variables sexo y edad}

Las mujeres mayores de 46 años evidencian rasgos de mayor conservadurismo y cumplen un rol importante en la preservación de la lengua originaria, con mayor preponderancia en el grupo nomatsigenga. Se observa que los jóvenes, hombres y mujeres ashaninka y yanesha se vinculan estrechamente con la lengua castellana, atribuyéndole características que facilitarían su integración a círculos sociales; por ello, no son agentes de conservación ni desarrollo de la lengua y cultura. Algo menos sintomático ocurre con los jóvenes nomatsigenga que, si bien ponderan la lengua castellana como sistema de mayor alcance social y educativo, se sienten afectivamente más involucrados con la lengua originaria.

A partir de la evaluación de los "pares ocultos", aquellos que tienen relación con el ámbito laboral y/o profesional, señalan que los pares de adjetivos puntuados favorecen a la voz castellana y que en el caso de los personales y afectivos favorece a la lengua originaria. Estos resultados son bastante equilibrados, para 
el caso de la variable edad, especialmente en el grupo etario de 31 a 45 años y de 46 a más años, pues no hay diferencia en puntuación sustantiva entre la lengua originaria (ashaninka, yanesha) y la producción en castellano. Para el caso de la población nomatsigenga, la mayoría de los tres grupos etarios - con mayor intensidad y proporción los de 46 a más años-, ponderan el uso de la lengua originaria, en algunos, incluso en el relativo a pares de adjetivos dicotómicos vinculados al ámbito laboral.

Si bien los jóvenes, y sobre todo los adultos de los grupos ashaninka y yanesha, están identificados con su lengua y cultura, hay una valoración y actitud positiva hacia el castellano, pues lo consideran un vehículo importante para el proceso educativo, la interacción social y comercial con el mundo exterior. El escenario de globalización en que vivimos también los involucra e influye sobre todo en los jóvenes que desplazan sistemáticamente a la lengua originaria en favor del castellano.

Podemos afirmar, entonces, que los jóvenes ashaninka y yanesha, en el marco de la cotidianidad, no usan la lengua más que esporádicamente al interior de contextos familiares. A pesar de que dicen estar orgullosos de su lengua y cultura, prefieren el uso del castellano; inclusive adoptan patrones culturales ajenos a la tradición propia. En el caso particular de algunos jóvenes ashaninka, cabe puntualizar el abandono absoluto de la lengua originaria y sobrevaloración del castellano como único instrumento de comunicación e interacción intracomunal, intercomunal y extracomunal.

En el caso de los jóvenes nomatsigenga, contrariamente, si bien asumen la importancia del uso del castellano, no evidencian matices marcados de desplazamiento de la lengua, sino parte del proceso comunicativo intergeneracional, sobre todo en la comunidad de Alto Anapati.

\subsubsection{Variable escolaridad}

En las comunidades ashaninka, yanesha y nomatsigenga predomina el uso de la lengua castellana en contextos institucionales. Esta es considerada como la más apropiada en el proceso educativo. En dicho marco, la intervención de profesores, 
mayormente foráneos, genera muchas veces un conflicto entre la visión occidental y la visión del mundo nativo; consecuentemente, se producen influencias en las actitudes lingüísticas de la población. Es evidente que estos elementos repercuten en el proceso de integración equilibrada en la sociedad mayor e inevitablemente la segregación y marginación, más allá de la lengua y cultura, del individuo con sus diferencias, con sus valores.

Las personas, independientemente del sexo, que han alcanzado un nivel de instrucción superior (contrario a las actitudes lingüísticas de algunos ashaninka) son más conscientes de la situación de uso poco favorable de su lengua originaria y la asumen afectivamente como parte de la población a la cual pertenecen. Las personas del grupo ashaninka y yanesha de nivel secundario o primario consideran que la lengua originaria se ha convertido en un obstáculo para efectos del desarrollo académico, laboral y para las posibilidades de interacción con agentes foráneos. En el caso de la población nomatsigenga que solo cuenta con una persona que tiene estudios superiores, la variable escolaridad evidencia marcada identificación con la lengua y cultura originaria. Se aprecia una actitud positiva y uso recurrente de la lengua nativa con matices poco consistentes a escala intracomunal de desplazamiento del nomatsigenga en favor del castellano.

\subsubsection{Variable procedencia}

Las poblaciones ashaninka y yanesha que tienen contacto permanente con agentes foráneos, en muchos casos, han incorporado a personas de procedencia andina. Hay algunas, como la comunidad ashaninka de Marankiari Bajo, que responden a una población mixta, es decir ashaninka y colonos, mayoritariamente andinos con una marcada influencia de la religión adventista. En ese contexto, se evidencia una acentuada influencia de la lengua y cultura ajenas a la suya y que cada vez adquiere mayor consistencia de uso y sobrevaloración en perjuicio de la lengua originaria. Este hecho, en muchos casos repercute, especialmente entre la población ashaninka, en actitudes de autodiscriminación.

Cabe señalar que el total de indígenas nomatsigenga encuestados reside desde su nacimiento y en dos casos desde más de cinco años en su comunidad de origen; 
es relevante anotar que la comunidad nativa de San Antonio de Sonomoro, en relación con la comunidad de Alto Anapati, establece contacto permanente con agentes sociales foráneos; así, comparativamente, la primera localidad presenta gradualmente menor intensidad de uso de la lengua originaria. En función de los rasgos relevantes, ambas comunidades evidencian mayor lealtad lingüística que sus pares ashaninka y yanesha. Especialmente en la comunidad de Alto Anapati se puede advertir mayor frecuencia de uso de la lengua entre los tres grupos etarios consignados en el estudio.

\section{Interpretación y discusión de resultados}

Los estudios sobre actitudes lingüísticas de los bilingües ashaninka, yanesha y nomatsigenga se encuentran en una etapa inicial debido, entre otras razones, a los cambios sociales que van experimentando estos pueblos, los cuales se expresan en la lengua o lenguas que emplean.

Se puede advertir la falta de identificación de los hablantes con respecto a la lengua originaria, pues en el marco de la cotidianidad se hace uso del castellano, en diferentes contextos, incluido el hogar, con excepción de los usuarios de la lengua nomatsigenga que evidencian mayor intensidad y frecuencia de uso en lengua originaria, especialmente en la comunidad de Alto Anapati.

De manera particular, en los hablantes de la lengua ashaninka, desde la dimensión afectiva, la orientación es hacia una relativa identificación con la lengua originaria y con las tradiciones; por tanto, se presenta una actitud positiva casi en términos igualitarios con el obtenido para el castellano. Sin embargo, desde la mirada cualitativa se puede señalar que de acuerdo con los datos recogidos se evidencia un abandono sistemático de la lengua originaria en favor del castellano, el mismo que desplaza parcial y hasta totalmente a la lengua nativa en algunos grupos etarios (jóvenes y niños). A pesar de los grandes cambios que experimenta el mundo, mantienen una serie de patrones tradicionales que los identifican como "nativos"; no obstante, cada uno de ellos, en mayor o menor medida, se halla en un inminente proceso de cambio sociocultural y lingüístico. 
Los datos recogidos a través del diferencial semántico circunscriben situaciones y motivaciones de acomodo de los colaboradores respecto de expectativas que pueden significar la promoción de la lengua originaria hacia el exterior, así como la acentuada afectividad y vínculo sentimental con la lengua originaria, fundamentalmente en el caso de los adultos (31-45 años) y los mayores (46 a más años). En esta perspectiva, la interpretación desde la óptica cualitativa permite evidenciar, fundamentalmente entre los jóvenes ashaninka y yanesha, la falta de identificación con la lengua originaria, pues en el marco de la cotidianidad se hace uso, en diferentes contextos, incluido el hogar, del castellano.

Este tipo de comportamientos no es privativo de los grupos ashaninka y yanesha, pues se observa características similares en el estudio de Hidalgo \& Pineda (2014, pp. 26-28) en el que se afirma la evidencia de una doble y contradictoria actitud de los pobladores ante la lengua indígena; una en tanto actitud de respeto y admiración cuando se les pregunta por ella de manera directa; y otra, cuando a través de pruebas encubiertas se expresa una actitud negativa sobre el uso de la lengua originaria. Estas características se pueden reconocer de manera particular y de acuerdo con los datos recogidos en las poblaciones ashaninka y yanesha. Así, en acciones cotidianas de estos sectores de la población, se evidencia un abandono sistemático de la lengua originaria en favor del castellano y una falta de identificación con la cosmovisión del grupo, proceso que se produce fundamentalmente entre los jóvenes y niños, aunque también parcialmente entre los adultos.

Otro de los factores que influye en el desplazamiento de la lengua amerindia y la proyección de uso absoluto de la lengua castellana es la interacción permanente que se establece con los poblados urbanos en desigualdad de condiciones sociales, culturales y económicas, esto es, influencia de actitudes negativas hacia la lengua originaria. Asimismo, las carreteras que fueran construidas con el fin de servir de nexo entre las comunidades nativas y el mundo externo para favorecer a las primeras, solo han provocado cambios socioculturales, lingüísticos y económicos desfavorables para la comunidad ashaninka y yanesha. En este marco cabe destacar el fenómeno de la migración iniciado hace unas décadas, el mismo 
que se ha intensificado en los últimos años llevándolos a trasladarse hacia áreas urbanas de regiones andinas y sobre todo costeñas, y Lima preferentemente.

Los cambios acelerados de tipo económico, cultural y social de las poblaciones ashaninka y yanesha, no son ajenos a los de las poblaciones amerindias amazónicas, pues son producto de los cambios que experimenta la sociedad hegemónica, los cuales se expresan en la preferencia por el castellano, actitud que va en desmedro de la lengua indígena, ya que las limita en sus funciones restringiéndolas a un ámbito doméstico (familiar), incluso, virtualmente, a la sustitución absoluta de la lengua originaria.

Es relevante anotar que las comunidades nativas en el valle del Perené, Palcazú y Pangoa establecen contactos permanentes y esporádicos con agentes sociales no nativos, dependiendo de su cercanía o lejanía a las vías más importantes de centros poblacionales; es decir, depende de la configuración sociocultural que presentan las comunidades indígenas. En el análisis inicial de cada una de las variables contempladas en el estudio, se puede caracterizar los factores que condicionan la predisposición valorativa de los usuarios indígenas amazónicos respecto a su lengua, al castellano y su identificación con el grupo y la sociedad en su conjunto. Los ashaninka del valle del Perené, desde el punto de vista etnohistórico tienen una trayectoria de contacto cada vez más intensa con el mundo exterior, ya sea con andinos, colonos, conquistadores, misioneros siempre en condiciones de desventaja, de sumisión forzada.

Si añadimos a la evaluación de variables contempladas en el estudio, la intervención de factores educativos, de contacto social inmediato con los mestizos, de modernización y de migración a las ciudades tanto andinas como costeñas (Lima), el futuro cercano es de paulatina pérdida de identidad lingüístico-cultural y, consecuentemente, del debilitamiento y proceso de extinción de las lenguas; además, cabe destacar la influencia significativa en el proceso de formación académica y personal del individuo indígena que posibilitará la continuidad de la marginación de la que han sido objeto a través de su historia, relegados a escalas más bajas dentro de la sociedad hegemónica. En el marco de los resultados de su investigación, González (2014) afirma que los usuarios de la lengua quechua de 
Abancay la consideran un instrumento de identificación y más el apropiado para expresarse en todos los contextos (González, 2014, pp. 8-10), a diferencia de los usuarios de las lenguas ashaninka y yanesha (en su mayoría jóvenes) que piensan que la más apropiada y de mayor utilidad es la lengua castellana. En la dimensión afectiva, se advierte un fuerte vínculo de la población nomatsigenga con la lengua y la cultura nativa; en menor grado, ocurre en la dimensión cognoscitiva, pues se pondera la lengua castellana en función de poder, utilidad, desarrollo laboral y académico.

Para las poblaciones ashaninka y yanesha, en ese orden, a excepción de la población adulta mayor, en particular, las mujeres, la orientación y valoración afectiva y cognoscitiva la tiene la lengua castellana, pues consideran que les facilita los procesos de interacción social, cultural y económica. En estas comunidades de habla, se evidencian rasgos identitarios de grupo débil pues la incursión del castellano y la cultura occidental en las actuales condiciones atentan con franco desplazamiento de las lenguas ashaninka y yanesha.

Por ello, consideramos la necesidad de implementar una política y planificación lingüística en la que previamente se contemple un diagnóstico actualizado que involucre aspectos educativos formales e informales de los grupos indígenas y no indígenas, sobre todo, respecto al conocimiento de la realidad lingüísticocultural del país y la importancia de esta en la configuración sociolingüística de la Amazonía; del mismo modo, aspectos sociales, culturales, ideológicos, legales, etc., que contribuyan al fortalecimiento de la autoestima del individuo ashaninka como persona, como depositario de una tradición, de una lengua, de una cultura.

Como casi todos los grupos indígenas, y por cierto los ashaninka, los yanesha y los nomatsigenga mantienen relaciones de interacción con miembros de la sociedad mayoritaria, es un imperativo la sensibilización de todos los agentes sociales, indígenas o no, de la existencia de sociedades amazónicas en situación de contacto lingüístico desde diferentes vías: educación formal e informal, local, regional y nacional, uso de la lengua indígena oral y escrita. 


\section{Conclusiones}

1. Las actitudes lingüísticas vinculadas con la identidad del grupo en las comunidades yanesha y ashaninka evidencian rasgos identitarios de grupo bastante débil. Los agentes más importantes en el abandono de la práctica lingüística y cultural son los jóvenes.

2. Las variables sexo y edad cuantitativamente no tienen mayor influencia en la valoración de las lenguas ashaninka, yanesha, nomatsigenga y castellana; sin embargo, cualitativamente permiten apreciar una mayor valoración cognoscitiva, sociocultural y afectiva hacia la lengua castellana, sobre todo en el grupo etario joven, con ligera predominancia de varones. Asimismo, la orientación etnocentrista de los jóvenes refleja una tendencia de abandono de las prácticas tradicionales y una actitud sistemática desfavorable hacia su lengua materna, especialmente en la población ashaninka y yanesha. Así, en el desarrollo de acciones cotidianas, los adultos (31 a 45 años) y en mayor medida los jóvenes (16 a 30 años) muestran actitudes positivas hacia la lengua castellana y, sistemáticamente negativas hacia la lengua originaria.

3. Los resultados obtenidos en el estudio coinciden con las conclusiones obtenidas en otros, donde se consideró la hipótesis del valor inherente, en la cual la población compara dos lenguas, valorando a una como más atractiva que la otra, y la hipótesis del valor impuesto, la cual valora una lengua determinada puesto que es utilizada por el grupo de mayor prestigio o poder. En este caso, la conciencia lingüística de la población bilingüe yanesha, ashaninka y nomatsigenga hace posible que diferencien a los sujetos de las grabaciones e identifiquen en algunos casos el lugar de procedencia de estos; aunque es posible que solo hagan la diferencia entre indígenas o no indígenas, en lugar de identificar al sujeto 2 como hablante de una lengua originaria específicamente. En este marco, se puede manifestar que la población encuestada sí pone de manifiesto la preferencia a la lengua castellana sobre la originaria, asignando características más positivas tanto en el aspecto social y personal, como 
aquellas cualidades que llevarían a un sujeto a una integración o aceptación en el ámbito laboral; en menor medida se evidencia en la población nomatsigenga.

4. Los ancianos de las diferentes comunidades, especialmente ashaninka y yanesha, han perdido la práctica tradicional, la visión del mundo propia de cada pueblo originario. Se debe destacar mayor consistencia y práctica de la lengua y tradición entre los pobladores nomatsigenga. En el caso ashaninka, en la comunidad de Bajo Chirani se puede afirmar que no existen ancianos con conocimientos tradicionales y menos de injerencia en la conservación de prácticas tradicionales como era común observar hasta hace unos 25 años. Consecuentemente, no ocurren procesos de comunicación intergeneracional que permitan la continuidad y desarrollo de algunas costumbres y más bien se intensifican actitudes lingüísticas negativas hacia la lengua originaria, dinamizando el empoderamiento de la cultura occidental y los rasgos de precarización de la identidad y autoestima del poblador ashaninka, y en menor medida yanesha.

5. Las variables escolaridad y procedencia cuantitativamente influyen de forma significativa en las actitudes lingüísticas de la población yanesha; cualitativamente se advierte la influencia de las dos lenguas mencionadas. En el caso ashaninka, la escolaridad en la comunidad de Bajo Chirani (procedencia) se percibe como de influencia negativa hacia la valoración de la lengua originaria; mientras que entre la población nomatsigenga, la influencia es todavía incipiente, pues responden a comunidades más conservadoras en comparación con las ashaninka y yanesha.

6. Es imperativo ampliar y profundizar la evaluación de los factores que condicionan las actitudes hacia las lenguas en otros grupos indígenas y no indígenas que a su vez sirvan de base para implementar los lineamientos de política y planificación lingüística y educativa en una perspectiva de valoración no solo de las lenguas y culturas amazónicas, sino del individuo indígena y la comprensión, tolerancia y respeto por parte de los no indígena dentro y fuera de las comunidades nativas. 


\section{Referencias bibliográficas}

Acheyisi, R. \& Fishman, J. (1970). Language attitudes studies. A brief survey of methodological approaches. Anthropological Linguistics, 12(5), 137157.

Alvar López, M. A. (1986). Hombre, etnia, estado: actitudes lingüisticas en Hispanoamérica. Madrid: Editorial Gredos.

Álvarez, A., Martínez, H., \& Urdaneta, L. (2001). Actitudes lingüísticas en Mérida y Maracaibo: Otra cara de la identidad. Boletín Antropológico, 52(2), 145-166.

Appel, R., \& Muysken, P. (1996). Bilingüismo y contacto de lenguas. Barcelona: Ariel.

Areiza, R., Cisneros, M., \& Tabares, L. (2012). Sociolingüística: enfoque pragmático y variacionista, segunda ed. Bogotá: Ecoe Ediciones.

Blas Arroyo, J. L. (2012). Sociolingüística del español. Madrid: Cátedra.

Carranza Romero F. (1982). Resultados lingüisticos del contacto quechua español. Lima: Concytec.

Castillo Hernández, M. A. (2007). Mismo mexicano pero diferente idioma: Identidades y actitudes lingüísticas en los maseualmej de Cuetzalan. Ciudad de México: Universidad Nacional Autónoma de México, Instituto de Investigaciones Antropológicas.

Falcón, Pedro \& Mamani, Luis (2017). Actitudes lingüísticas en contextos interculturales: población asháninka Bajo Chirani. RLA. Revista de lingüistica teórica y aplicada. http://dx.doi.org/10.4067/S071848832017000100095

Fasold, R. W. (1996). La sociolingüistica de la sociedad: introducción a la sociolingüistica. Madrid: Visor libros.

Fishbein, M. (1965). A consideration of beliefs, attitudes, and their relationships. En I. D. Steiner \& M. Fishbein, Eds. Current Studies in Social Psychology (pp. 107-120). Nueva York: Holt, Rinehart \& Winston.

García Marcos, F. (2015). Sociolingüistica. Madrid: Editorial Síntesis.

Gómez Molina, J. R. (1998). Actitudes lingüisticas en una comunidad bilingüe y multicultural. Valencia: Universitat de València. 
González, D. (2014). Un estudio de actitudes hacia el quechua del este de Apurímac. SIL International. Recuperado de https://www.sil.org/system/ files/reapdata/10/37/75/.../silesr2014_001.pdf.

Hernández, R.; Fernández, C. \& Baptista, P. (2014). Metodología de la Investigación ( $6^{\mathrm{a}}$. ed.). Ciudad de México: McGraw Hill.

Hidalgo Martínez, M. \& Pineda Cruz, A. (2014). La lengua indígena como factor de discriminación en dos comunidades de Guerrero. Fuentes Humanisticas, 28(49), 13-34.

Howard, R. (2007). Por los linderos de la lengua. Lima: Instituto Francés de Estudios Andinos, Instituto de Estudios Peruanos, Fondo Editorial de la Pontificia Universidad Católica del Perú.

López Morales, H. (2015). Sociolingüistica. Madrid: Gredos.

Moreno Fernández, M. (2009). Principios de sociolingüística y sociología del lenguaje, cuarta ed. Barcelona: Ariel.

Sarnoff, I. (1966). Social attitudes and the resolution of motivational conflict. En M. Jahoda \& N. Warren (Eds). Attitudes (pp. 271-282). Harmondsworth: Penguin.

Silva-Corvalán, C. (2001). Sociolingüística y pragmática del español. Washington, D. C.: Georgetown University Press.

Street, R. L. \& Hopper, R. (1982). A model of speech style evaluation. En H. Giles y E.B. Ryan (eds). Attitudes toward Language Variation (pp. 175188). Londres: Arnold Publishers.

Trudell, B. (2008) Más allá del aula bilingüe, segunda ed. Lima: Ministerio de Educación, Instituto Lingüístico de Verano.

Vargas, G. (2017). Validez y confiabilidad de la escala de actitudes hacia el reciclaje y uso responsable de papel en los estudiantes de la UNMSM. Revista Letras, 88(128), 207-217. 